\title{
Some Problems of Solar Quiescent Prominences in the Light of Ambartsumian's Ideas
}

\author{
A.Nikoghossian \\ NAS RA V. Ambartsumian Byurakan Astrophysical Observatory (BAO), Armenia \\ E-mail: nikoghoss@yahoo.com
}

\begin{abstract}
The report is a brief overview of our results in the theory of spectral line formation in atmospheres with complex fine structure. The research motivation was due to interpretation of the EUV spectrum of solar quiescent prominences observed with the SUMER spectrograph as a part of the SOHO space program. We describe the methods proposed for solving the line-radiation transfer problems in multicomponent atmospheres which use Ambartsumian's ideas proposed in the theory of radiation transfer and its applications. It is demonstrated that even the simplest static model radiating medium composed of physically different types of elements shows line profiles differing from those formed in the medium with preliminarily averaged properties. The more realistic and complicated case of a multicomponent atmosphere with randomly varying properties is considered with special attention paid to the effect of the velocity field. An important role of the relative mean square deviation of observed line intensities in the diagnostics of physical features of spatial and temporal variations of prominences is shown.
\end{abstract}

\section{Introduction}

The need to create a theory of radiation transfer in the media with complicated fine structure subject to random variations has long been felt in the theory of the solar atmosphere. As early as in the 1970' s Cram showed that the observed profiles of chromospheric lines of ionized calcium cannot be explained in terms of the theory developed for homogeneous stationary media. Similar problems arise also in interpreting nonstationary phenomena in spicules, coronal streams, and polar plums. Problems involving the propagation of radiation in randomly varying inhomogeneous media appear also in other branches of astrophysics. Examples include the scattering of light on molecular clouds in the interstellar medium (Juvela 1997, Juvela 
\& Padovan 2001) and the stochastic attenuation of galactic radiation as it travels over cosmologic distances (Madau 1995).

The procedure for calculating the statistical properties of the radiation emerging from a multicomponent LTE-atmosphere was presented by Lindsey and Jefferies (1987) and Lindsey (1988) for the interpretation of total solar eclipse observations in the far infrared. These authors derived relations describing the evolution behavior of the mean value of the intensity and its variance along the radiation path.

The theory we developed for interpreting the observable emergence of complex structural pattern of media in the line spectrum may be regarded as a further generalization of the Ambartsumian layers adding method (Ambartsumian 1944, 1960).

The theory is especially simple for the atmosphere in LTE: because of the absence of scattering, and hence reflection from structural elements, the mean value of intensity emerging from any part of the multicomponent atmosphere remains unaltered when new components are added. This fact is essential in the sense that it simplifies the problem and allows one to establish immediately the recurrence relationship for both the mean intensity and variance so that the problem admits a simple closed-form solution. These results for the LTE atmospheres were generalized by Nikoghossian and coauthors (Nikoghossian, Pojoga \& Mouradian 1997, 1999), which allows to reveal quantitative as well as qualitative discrepancies with respect to those formed in a homogeneous atmosphere with preliminarily averaged random characteristics. The further development of the theory given in (Nikoghossian \& Mouradian 2000) allows to formulate and solve the problem of the spectral line-radiation also in the NLTE composite atmospheres.

On the other hand, one should take into account the fact that the plasma in the prominences is known to participate in various kinds of motions which, of course, affect the observed spectral line profiles. Random thermal and turbulent motions in prominences with velocities on the order of $5-10 \mathrm{~km} / \mathrm{s}$ are superimposed on ascending (eruption) or descending (mass loss owing to its return to the chromosphere and photosphere) flows and large-scale hydrodynamic motions (Tandberg-Hanssen 1995). The radiation transfer in a line through a dynamically active medium of this sort leads to a great variety of line shapes which are distorted by the Doppler effect. Furthermore, the line profiles depend on a number of other parameters characterizing the emitting volume, so that a quantitative interpretation of the observed spectra is a rather complicated problem in general. This more realistic problem making allowance for the dynamics of a radiating multicomponent medium was considered by the author in (Nikoghossian 2002). An important place in all the mentioned model problems occupies the frequency-dependent relative mean square deviations (RelMSD) of the observed line intensities due to spatial or/and temporal variation of the number or physical properties and non-thermal motions of the fine structural elements. The idea to use 
the variance of fluctuations in the observed superficial brightness as an additional tool in investigating astrophysical objects goes back to Ambartsumian's work (1944), where the brightness fluctuations of the Milky Way were used in estimating the value of interstellar absorption. Generalizing this approach, we showed that there is a well pronounced relationship between functional form of the RelMSD within a spectral line and the sources of the observed line intensity spatial/temporal fluctuations. The direct motivation of the theory we developed was the rich observational material gained in frameworks of the Skylab and SOHO space missions for the EUV spectrum of prominences. The theory we developed in the aforementioned papers is applied to find out some physical and geometrical features of solar quiescent prominences characterized by composite threadlike structure.

\section{Lines profiles in stochastic multicomponent me- dia}

We start with treating the simplest model of the static LTE multicomponent medium composed of a certain number of structural elements of two sorts each of which is characterized by its optical thickness $\tau_{i}$, the power of the contained energy sources $B_{i}$ and the probability $p_{i}$ of appearance (i $=1,2)$. The dependence on frequency within the spectral line is described by the Dopplerian profile of the absorption coefficient $\alpha(x)=\exp \left(-x^{2}\right)$, where the dimensionless frequency $x$ is measured from the center of the line in Doppler widths. We are interested with the statistical mean value of the observed intensity $\left\langle(I(x)\rangle\right.$ and the RelMSD $\delta_{N}(x)$ in the line, where $N$ denotes the number of structural elements. The closed form analytical expressions for the mentioned quantities were obtained in (Nikoghossian, Pojoga \& Mouradian 1997, 1999)

$$
\begin{gathered}
\left\langle I_{N}(x)\right\rangle=L_{N}(x)\left\langle I_{1}(x)\right\rangle, \\
\delta_{N}(x)=\frac{M_{N}(x)}{L_{N}(x)}\left[1+\delta_{1}(x)\right]+2 \frac{K(x) A_{N}(x)}{\left\langle I_{1}(x)\right\rangle L_{N}^{2}(x)}-1,
\end{gathered}
$$

where the following notations are introduced

$$
\begin{gathered}
L_{N}(x)=\frac{1-q^{N}(x)}{1-q(x)}, \quad M_{N}(x)=\frac{1-\beta^{N}(x)}{1-\beta(x)}, \quad A_{N}(x)=\frac{L_{N}(x)-M_{N}(x)}{q(x)-\beta(x)}, \\
K(x)=\left\langle J_{i}(x) e^{-\alpha(x) \tau_{i}}\right\rangle, \quad q(x)=\left\langle e^{-\alpha(x) \tau_{i}}\right\rangle, \quad \beta(x)=\left\langle e^{-2 \alpha(x) \tau_{i}}\right\rangle, \\
J_{i}(x)=B_{i} \alpha(x)\left[1-e^{-\alpha(x) \tau_{i}}\right],
\end{gathered}
$$



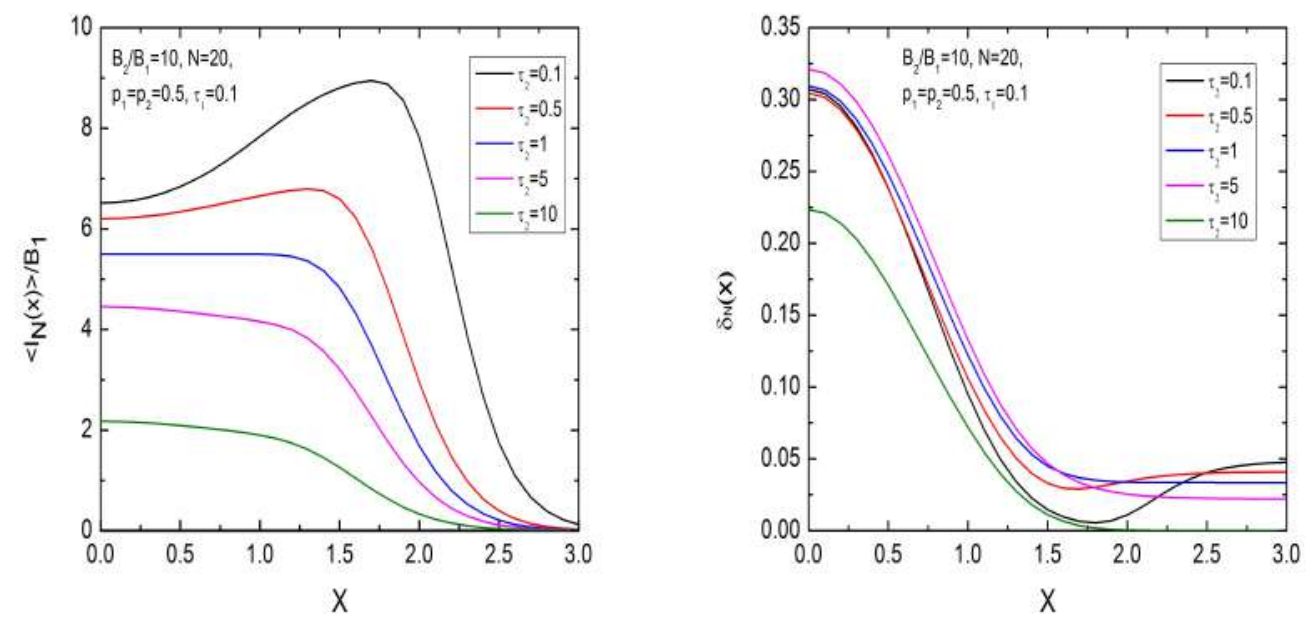

Figure 1: The statistical mean of the emitting intensities (left panel) and RelMSD (right panel) for different values of parameters. Special attention is payed to different combinations of optical thicknesses of structural elements

According to definition, we have

$$
\left\langle I_{1}(x)\right\rangle=\sum_{i=1}^{2} p_{i} J_{i}(x), \quad \delta_{i}(x)=\frac{1}{2\left\langle I_{1}(x)\right\rangle^{2}} \sum_{k=1}^{2} p_{i} \sum_{i=1}^{2} p_{i}\left[J_{i}(x)-J_{k}(x)\right]^{2},
$$

We see that the degree of fluctuations for any number of components $N$ is determined by four parameters $q, \beta, \delta_{1}$ and $K /\left\langle I_{1}\right\rangle$ (or by three parameters for $p_{1}=p_{2}$ ), which are statistical characteristics of the emission and absorption of a single element of the multicomponent medium.

Fig.1 illustrates profiles of spectral lines and proper RelMSD when the number structural elements is 20. In this model case the elements differ with each other in the power of the internal energy sources. The opacities are choosen on the ground that the hotter components must mainly be more opaque. The first effect that attract attention is the double-peaked profile formed preferably in cases when difference between components are due predominatly to the value of the sources of internal energy. Remind that such profiles are usually associated with the lines formed in a NLTE atmosphere as a result of the frequency redistribution. We see now that multicomponent media may generate double-peaked profiles even in the abscense of the radiation scattering. Another result concerns the frequency behaviour of the RelMSD in the wings of the line. It is also seen that its value can either decrease or increase dependent on values of other parameters.

Further progress of the theory allowed to handle more general and complex models with greater sorts of components and greater number of possible 

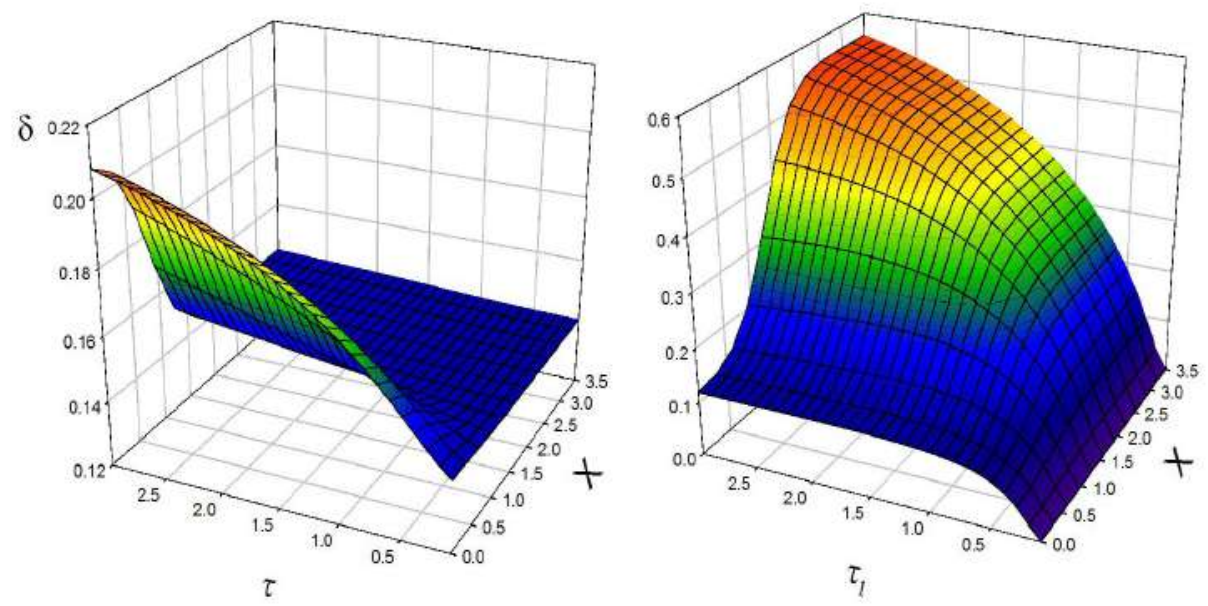

Figure 2: The RelMSD of intensity fluctuations in two models of the NLTE atmosphere. Calculations are performed for $\lambda=0.99$, where $\lambda$ is the photon re-radiation coefficient in the multiple scattering process. For simplicity's sake, the number of components are taken $N=4$ and $p_{1}=p_{2}$. The values of other parameters are $S=B_{2} / B_{1}=7, \tau_{1}=\tau_{2}=\tau$ (left panel) and $S=1$, $\tau=0.1$ (right panel)

realizations. More realistic models consider the correlations between realizations of different parameters in two adjacent components. The theory becomes much more complicated in the case of NLTE atmospheres (see the above cited papers of the author). The most important conclusion is that the aforementioned features of RelMSD remain qualitatively valid in the NLTE media. Fig.2 shows the frequency-dependent RelMSD of observed intensities in two mutually opposite situations of equal opacities $\tau_{1}=\tau_{2}$ and $B_{1} \neq B_{2}$ and the opposite case of differring opacities and equal $B_{i}$.

We see the quite distinct variation of the RelMSD over the entire frequency range within a line. This effect can obviously be regarded as a diagnostic tool in revealing the physical properties of various regions of prominences. It is evident that the real state of the prominence is generally more complex and depends on variations of both the mentioned parameters. In this more realistic situation when the optical thickness in the line and the power of the energy sources take arbitrary non-equal values, the variation of the RelMSD towards the wings of a line becomes more complex (Nikoghossian 2002, Nikoghossian, Aboudarham \& Mouradian 2005). The resulting center-to-wings variation of the RelMSD obviously depends on that which of considered causes of fluctuations predominates (see Fig,3). The situation becomes simpler if we suppose that the opaque lines are formed in the hotter radiating volumes, which is often the case. It must be noted that 

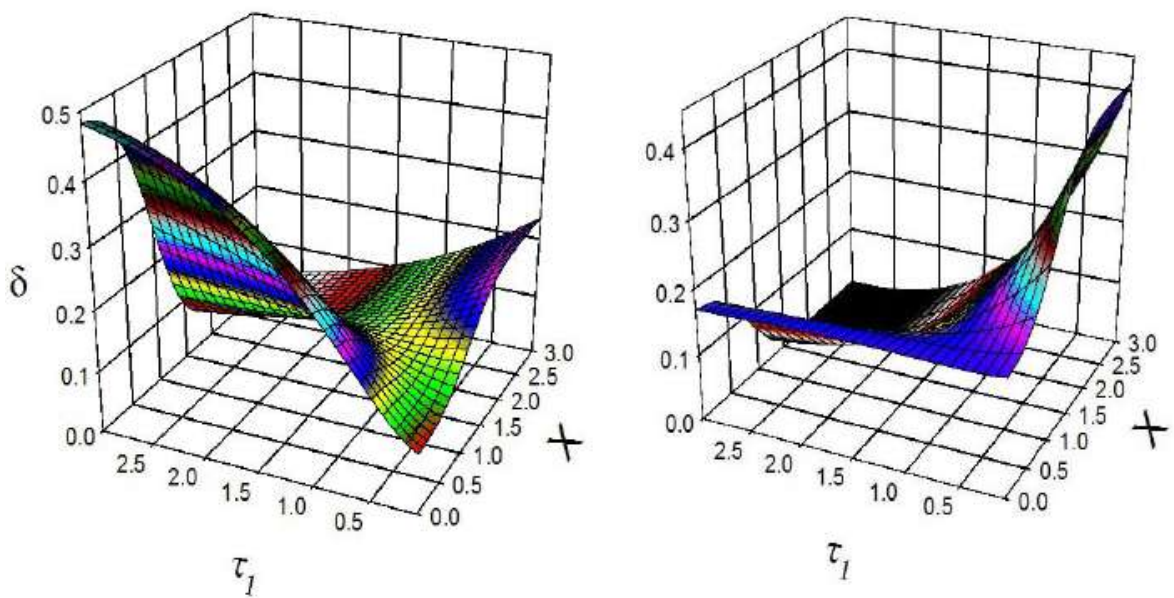

Figure 3: The RelMSD of intensity fluctuations in two model atmospheres for $N=4$ and $p_{1}=p_{2} . S=B_{2} / B_{1}=7, \tau_{2}=1: \lambda=0$ (LTE,left panel) and $\lambda=0.99$ (Non-LTE,right panel)

these effects quantitatively depend on the number of structural elements. Fig, 4 illustrates the variation of averaged values of dimensionless intensities and proper RelMSD in frequency within a line. As might be expected, with growing the number of components, the values of RelMSD decrease towards to wings. As above, the behavior of the RelMSD in the far wings of the line in these two model cases is diametrically different. It is not worthy that these considerations are important from the point of view of interpretation of the nature of the surface brightness fluctuations of any extended astronomical object with a randomly varying fine structure.

\section{Lines profiles in dynamically active multicompo- nent media}

As it was mentioned, in the real stochastic atmospheres of astrophysical interest the velocity field is subject to random variations together with physical and geometrical characteristics of the medium. It is evident that such kind of velocity variations may affect the observed line profiles generally in a great degree. Radiative transfer in a line through a dynamically active medium leads to a great variety of line shapes which are distorted by the Doppler effect. Furthermore, the line profiles depend on a number of other parameters characterizing the emitting volume, so that a quantitative interpretation of the observed spectra is a rather complicated problem, in general. The problem of the spectral line formation in a stochastic multicomponent atmosphere in LTE was treated by the author in (Nikoghossian, 

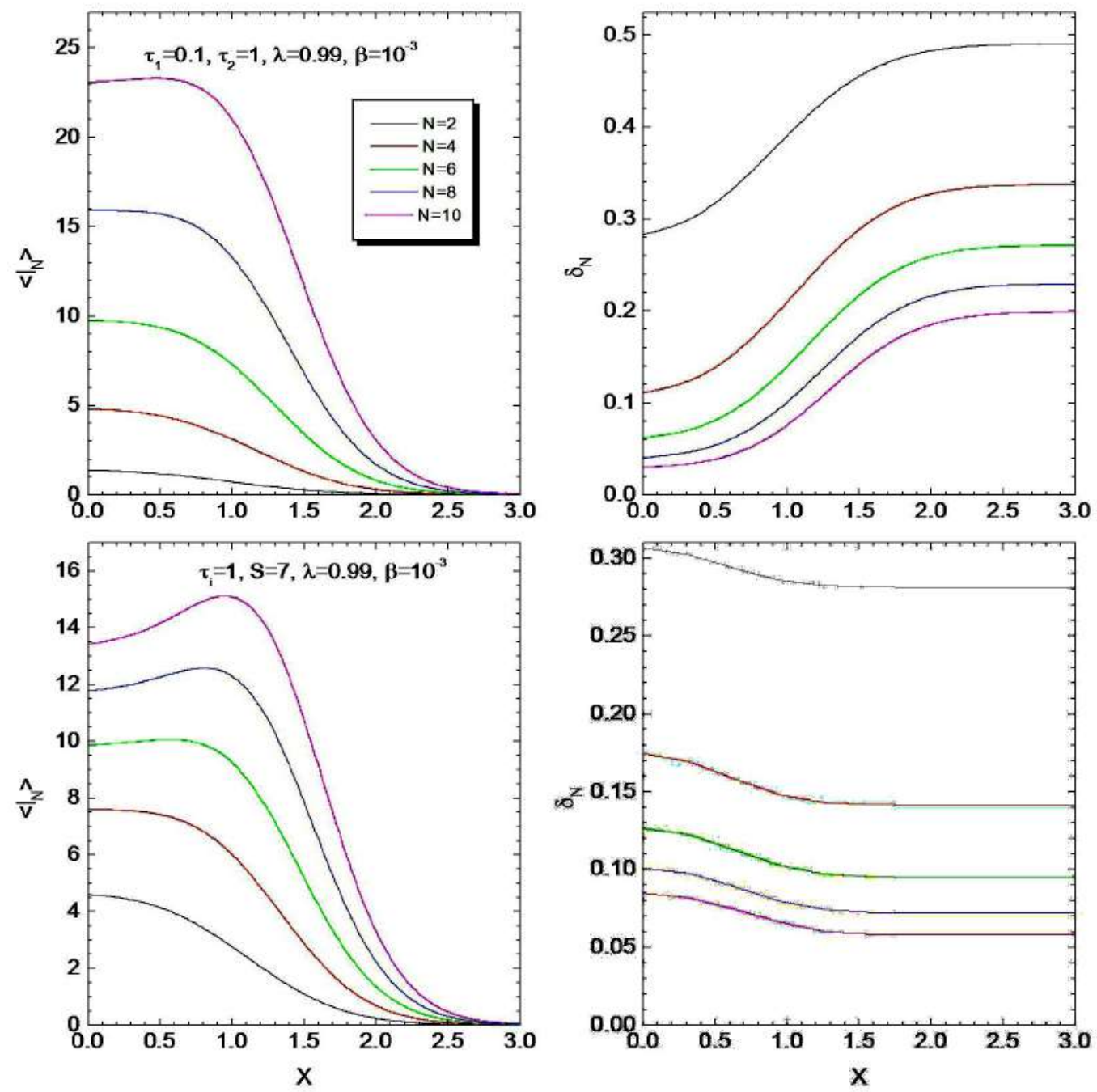

Figure 4: Dependence of averaged intensities radiating in a line and the proper RelMSD on the number of structural elements for indicated values of realized opacities, the power of internal energy sources and other parameters describing the elementary event of scattering ( $\beta$ is the ratio of the absorption coefficient in continuum to that in the centre of the line) 
2007). Motions considered in this paper imitate the large scale hydrodynamic motions usually observed in solar prominences. The effect of the radiation on the velocity field is neglected which is reasonable for the lines with low optical thickness. We set ourselves the problem of determining the radiation emerging from a dynamically active atmosphere consisting of $N$ structural elements whose physical characteristics undergo random variations. It is assumed that the spatial process of realization of components of a given type is Markovian, i.e., the realization of physical properties of a given component depends only on the realized properties of the preceding component. Particular attention we paid to RelMSD of the intensity of the observed radiation. The important feature of the developed method is that, for determining the mean intensity of the emerged line radiation, $\left\langle I_{N}(x)\right\rangle$, one preliminarily finds the auxiliary quantities $\left\langle I_{N}^{i}\left(x_{i}\right)\right\rangle$ characterizing the mean mathematical expectation of the intensity of the observed radiation under the condition that the $N$-th element belongs to the $i$-th type, so that

$$
\left\langle I_{N}(x)\right\rangle=\frac{1}{n} \sum_{i=1}^{n}\left\langle I_{N}^{i}(x)\right\rangle
$$

In the case of Markov process $\left\langle I_{N}^{i}(x)\right\rangle$ are determined by reccurence relations

$$
\left\langle I_{N}^{i}(x)\right\rangle=\sum_{k=1}^{n} q_{i k}\left[\left\langle I_{N}^{i}(x)\right\rangle \exp \left(-z^{i} \tau_{i}\right)+P_{N-1}^{k} J_{i}(x)\right],
$$

where the following notations are introduced: the coefficients $q_{i k}$ are elements of the Markov transition matrix, $z^{i}=\alpha\left(x-u^{\prime}\right)+\beta$ and $J_{i}(x)=$ $B_{i}\left[1-\exp \left(-z^{i} \tau_{i}\right)\right]$, the $P_{N}^{i}$ represents the probability of an event in which an element of $i$-th type will appear at the $N$-th position. Further, $u^{i}=$ $V_{h y d}^{i} / V_{t h}$, where $V_{t h}$ is the mean thermal velocity and $V_{h y d}$ - the line-of-sight component of the hydrodynamic velocity.

Fig.5 shows typical examples of profiles and RelMSD calculated for the purely stochastic case, when the atmosphere consists of only two types of structural elements. Along with the profile of the average radiation intensity, $\langle I\rangle$, emerging from the medium, we present two other profiles $\left\langle I_{1}\right\rangle$ and $\left\langle I_{2}\right\rangle$ calculated under the condition that the $N$-th element belongs, respectively, to the first and second types. To make the changes in the RelMSD clearer, $N$ has been chosen to be relatively small. In order to clarify the effect of the Markovian interaction between the components on observed profiles and the RelMSD, we considered the case of the so-called doubly stochastic atmosphere.

The profiles depicted in the right panel demonstrate essential distortion of the line profiles caused by hydrodynamic motions. Another important result is the distinctive feature of the RelMSD of the radiation in a line formed in a dynamically active stochastic atmosphere is local spikes (maxima) in 

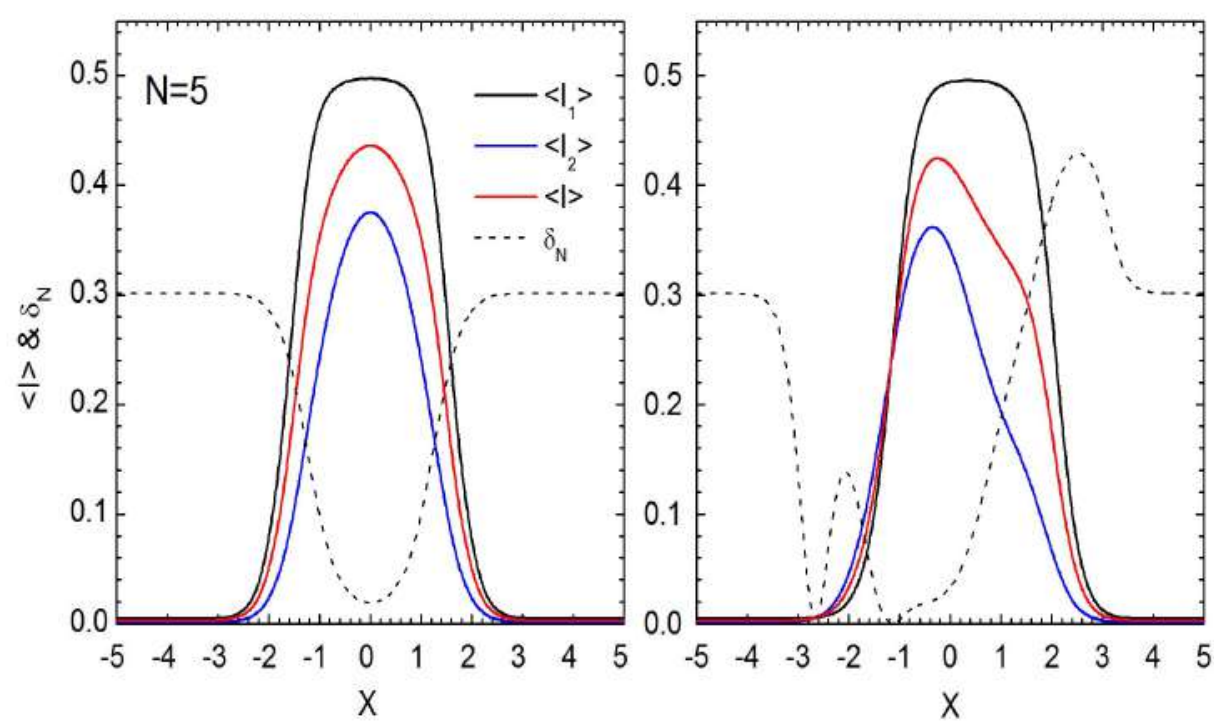

Figure 5: Theoretical profiles of a spectral line and the RelMSD for double stochastic static (left) and dynamically active (right) atmospheres. It is assumed that the latter consists of two types of structural elements with the following parameters: $B_{1}=B_{2}=1, p_{1}=p_{2}=0.5, \tau_{1}=2, \tau_{2}=0.2$, $\beta=10^{-3}, u^{1}=0.5, u^{2}=0.5$, and $q_{11}=q_{22}=0.9, q_{12}=q_{21}=0 . .1$

the wings of the line. The obtained theoretical results were compared with spectral observations of quiescent solar prominences obtained in framework of the SOHO space mission.

\section{Observation data}

In order to verify the theoretical results, we have used some observational data on spectral lines of solar quiescent prominences in the EUV domain of the spectrum obtained on August 10, 1999 using the SUMER spectrometer in frameworks of the SOHO space mission. We were interested in the fluctuations in the surface brightness of the prominences in several of strongest lines. With each observation, the spectrometer slit with its field of 1"120" yields 120 line profiles so that their variation along the slit could be traced. These data are usually sufficient for determining the statistical averages of the radiation intensity and the corresponding RelMSD as functions of frequency within a line.

Fig. 6 shows data on the relative mean intensity and RelMSD (broken curves) for several relatively strong EUV lines. We pointed out above that the RelMSD increases on going to the line wings. According to the theoret- 

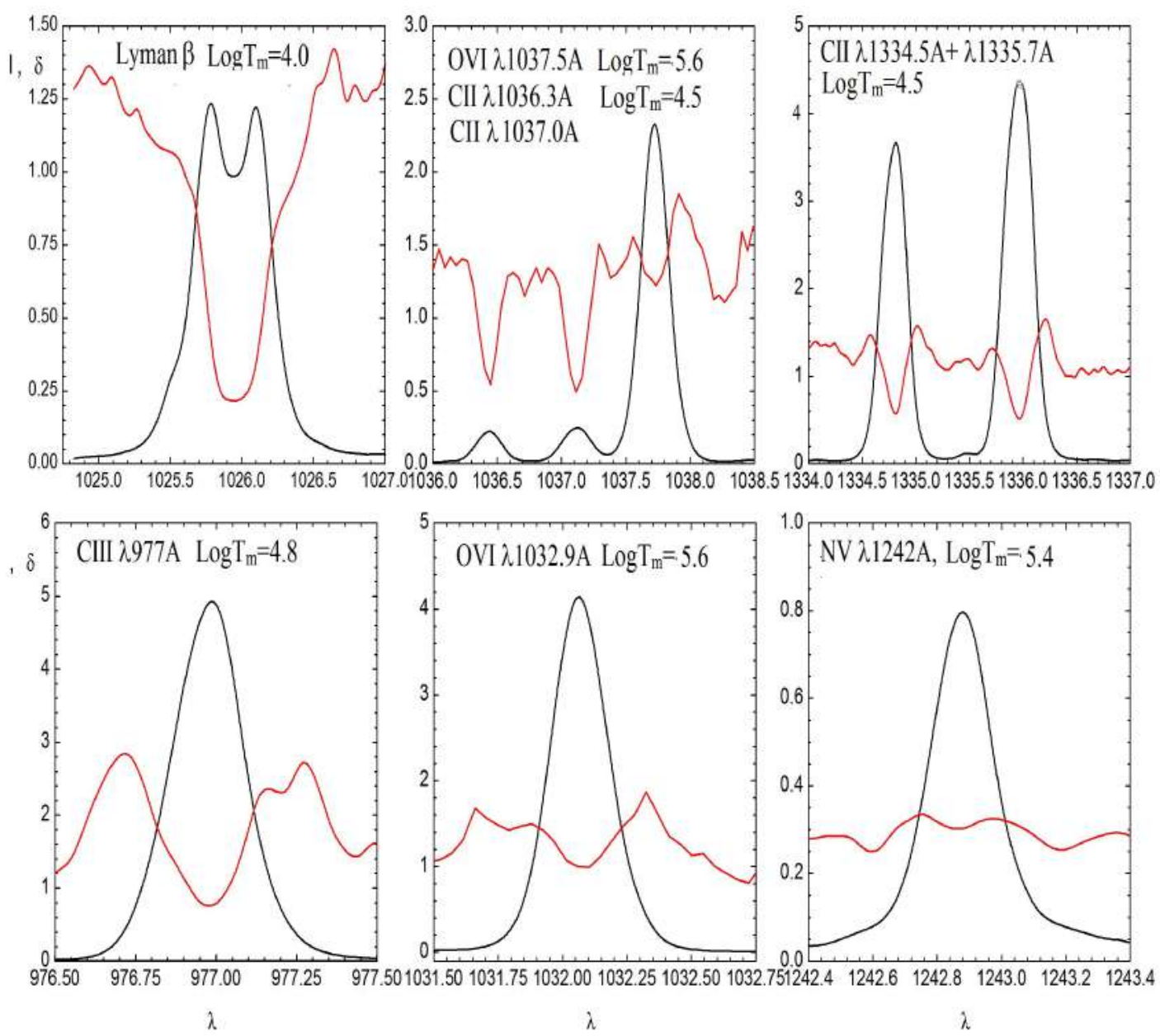

Figure 6: Statistical mean profiles (smooth curves) and the corresponding RelMSD (dotted curves) for a series of relatively strong EUV lines of a solar prominence observed on August 10, 1999, in frameworks of the SOHO space mission 
ical discussion, this was an indication of the fact that the fluctuations in the surface brightness of the prominence are caused mainly by changes in optical thicknesses. We see that the central depression in the frequency profile of the RelMSD is different for different lines and is correlated with the effective temperature at which they are formed. The spikes (local maxima) in the wings of the lines predicted by the above theory for non-thermal (hydrodynamic) motions can be distinctly seen. One can also see that for lines with a high effective formation temperature (i.e., the lines formed primarily in the surrounding corona), such as OVI $\lambda 1032 \AA, \lambda 1037 \AA$, and NV $\lambda 1242 \AA$, the magnitude of these spikes appears to be smaller than for the lines formed entirely within the prominence. The appearance of isolated single spikes is an indication that the velocities of the non-thermal motions are only slightly greater than the thermal speed, if they exceed it at all.

\section{Discussion}

The idea of invoking fluctuations in the surface brightness of prominences as an additional source of information on the radiating volume described above shows that important information can be extracted by studying the RelMSD of these changes as a function of frequency within a line. Theoretical studies have been compared with the data on changes in the profiles of EUV lines along the slit of the SUMER spectrograph obtained as part of the SOHO space program. Thus, it has been shown that, with some exceptions, fluctuations in the surface brightness of prominences in different lines are caused by changes in the optical thickness and that the changes are smaller when the lines are formed at higher effective temperatures. The study of these fluctuations is especially informative when large scale or hydrodynamic motions are present in the medium. If the radiating volume participates in rising and falling motions, then the frequency dependence of the RelMSD is characterized by unique spikes (local maxima) in the wings of the lines. The magnitude of the latter depends on the velocity of the large scale motion

\section{References}

Ambartsumian, V.A. 1944, Izv. AN ArmSSR, N1-2

Ambartsumian, V.A. 1944, Dokl. Acad. Sci. USSR, 44, 244

Ambartsumian, V.A. 1960, Scientific Works, v.1, Izd. AN ArmSSR, Yerevan

Cram, L.E. 1972, Sol, Phys., 22, 375

Jefferies, J.T.; Lindsey, C. 1988, Astrophys. J, 335, 372

Juvela, M. 1997, A\&A, 1997, 322, 943

Juvela, M.; Padovan, P. 2001, A\&A, 397, 201

Lindsey, C. 1987, ApJ., 320, 893 
Madau, P. 1995, ApJ. 441, 18

Nikoghossian, A.G. 2002, Astrophysics, 45, 223

Nikoghossian, A.G. 2007, Astrophysics., 50, 94

Nikoghossian, A.G.; Aboudarham, J., Mouradian, Z. 2005, Astrophysics. 48,253

Nikoghossian, A.G.; Mouradian Z. 2000, A\&A, 360, 2000

Nikoghossian, A.G.; Pojoga, S.; Mouradian, Z. 1997, A\&A, 325, 813

Nikoghossian, A.G.; Pojoga, S.; Mouradian, Z. 1999, A\&A, 342, 785

Tandberg-Hanssen, E. 1995, The Nature of Solar Prominences, Kluver, Dordrecht 